\title{
Chromatic Improvement of Backgrounds Images Captured with Environmental Pollution Using Retinex Model
}

\author{
Mario Dehesa, Alberto J. Rosales, Francisco J. Gallegos, Samuel Souverville, \\ and Isabel V. Hernández \\ Instituto Politécnico Nacional, ESIME Zacatenco, Ciudad de México, \\ Mexico \\ m_dehesa@hotmail.com, arosaless@ipn.mx
}

\begin{abstract}
A commonly problem of digital image processing systems that use video cameras for control navigation, as those used in cars or planes control, is that these systems depend on image contrast and the environmental pollution as fog, smog or rain. These environment characteristics, filters wavelengths of the light, which causes that the captured images, were modified by the video camera, decreasing its efficiency. It is proposed to improve the chromatic content of captured images, where environmental pollution is present, using the Retinex model. This algorithm implementation uses different characteristics such as lightness changes and color contrast; these characteristics produce different results for every Retinex model proposed showing differences in color and luminance modification of the captured image. In this paper are proposed and compared three different Retinex models; these models are the Simple Retinex, the Multi-scale Retinex and the Multi-scale Retinex with Color Correction.
\end{abstract}

Keywords: Retinex, contrast, lightness, color

\section{$1 \quad$ Introduction}

To be able to recognize license plates, face recognition, navigation systems, etc., it is important to have an acceptable visibility Rank, so the different components of an image could be observed. However, in real conditions, the clearness of the captured image is variable, where the conditions of the atmospheric air were pure, unfortunately the quality of the air changes from one place to another, and from one time moment to another, even if it is taken in the same place. Captured images in extreme conditions, where no visibility exist, because different conditions like dense fog, huge dust content caused by combustion materials, remains suspended in low layers of atmosphere, and does not allow maximum visibility.

The Retinex model, proposed by Dr. E. Land, has the characteristic to emulate the Human Vision System (HVS) behavior, and reproduce the color constancy phenome- 
non also called chromatic adaptation. Because of this phenomenon, is possible to identify that the actual color of an object does not affect the lightning color source [1], for example, if a space is illuminated for a tungsten filament bulb, the light that illuminates the object is warm light, however the objects inside the illuminated space retain their color balance. The similar behavior happen if the space is illuminated using a cold light source like green, blue, etc. This phenomenon is described by the Dr. Edwin H. Land. His research investigation explains how HVS understand colors and still today is kept under scientific research with many applications in fields like medical radiography, underwater photography, forensic photography, etc. [2].

In this research work, are used the methods of the Simple Retinex, the Multi-scale Retinex and the Multi-scale Retinex with Color Correction algorithms to prove and compare them as an improvement chromaticity method of the captured images.

\section{Retinex Models}

Were extracted the images used to compare different implementations of the Retinex algorithms from different digitizing media, like photographic cameras, cellphones or video cameras, all of this using weather environmental control.

Retinex models use mathematic operators resembling the color constancy phenomenon of the HVS. Color constancy is a desirable phenomenon in digitizing images, in order to identify the actual object color no matters lightning source characteristics. For this, there are different ways that improve the Retinex model [3].

The Retinex models receive as input, the magnitude values of the Red, Green and Blue channels to make an estimation of reflectance for each one, this has the purpose of being able to identify the lighting source, which allows knowing the intensity present in each pixel of the digitized image.

\subsection{Color Constancy}

Color constancy is a mechanism that allows HVS to identify the actual color of an object no matters the lightning source color, for example, from photometric point of view, a red object under a green light source, should produce the same spectral distribution as a green object under a red light source. Even in this condition, the object color remains stable regardless light source wavelength changes [4].

This phenomenon separates the light reflectance from light source, which can define the spectral illumination characteristics and makes an approximation of the reflectance value. The color constancy seen from HVS perception use space and chromatic resolution of each pixel to define scene appearance [5]. The following explains each Retinex applied suggested models.

\subsection{Simple Retinex}

Simple Retinex algorithm improves digitized captured images in low lightness conditions, using the equation (1). This algorithm imitates the chromaticity and local adaptability lightness for each pixel to get an approach of actual colors in a scene. 


$$
L=1 / N \sum_{i=1}^{N}\left(\log I\left(x_{p}\right)-\log \left(I\left(x_{i}\right)\right)\right),
$$

where $L$ represents the lightness of a pixel $\left(x_{p}\right)$, influenced by $N$ pixels $\left(x_{i}\right)$, where $i=$ $1,2,3 \ldots, N$, which will be selected in a random path. This process applies for every color channel of the RGB color space [6].

\subsection{Multi-scale Retinex (MSR)}

This model allows color identification in a more dynamic way because of the algorithm reduces abrupt illumination changes. The MSR model proposed by D. Jobson [4] is defined by the equation (2),

$$
R_{M S R_{i}}=\sum_{n=1}^{N} \omega_{n} R_{n_{i}}=\sum_{n=1}^{M} \omega_{n}\left[\log I_{i}(\mathrm{x}, \mathrm{y})-\log \left(F_{n}(x, y) * I_{i}(x, y)\right)\right],
$$

where $I=\mathrm{R}, \mathrm{G}$, and B channels. $M$ is the scales number, $\omega_{n}$ is a scale weight associated to an involving function $F_{n}(x, y)$ (Eq. (3)), * express convolution, $(x, y)$ are the coordinates of the pixels, $I_{i}$ is the actual image and is computed using the equation (4), $R_{M S R_{i}}$ is the output of the process,

$$
\begin{gathered}
F_{n}(x, y)=\mathrm{C}_{n} \exp \left[-\left(x^{2}+y^{2}\right) / 2 \sigma_{k}^{2}\right], \\
I_{i}(x, y)=\mathrm{S}_{i}(x, y) \mathrm{r}_{i}(x, y),
\end{gathered}
$$

where $\sigma_{k}$ is the typical standard deviation of the Gaussian envelope and its magnitude controls the envelope extension and the whole function is normalized as $C_{n}$ such that $\int F(x, y) d x d y=1, S_{i}$ represents the illumination and $\mathrm{r}_{i}$ is the scene reflectance.

\subsection{Multi-scale with Color Correction (MSRCR)}

The color theory investigation has generated different opinions about the nature of the color image components, one of this is known as - grey world-, this theory assumes that the average value of color variations that comprises the image should average a gray tone in common [7]. In images where is found a dominant color, the MSRCR method can give a result of gray image due to low saturation color. To correct the dominant color delivered for the MSRCR method, modifies the output of the MSR model, multiplying the dominant color for an image chromaticity restoration function [4]. The first step is to calculate chromaticity as shown in equation (5):

$$
I^{\prime}{ }_{i}(x, y)=\frac{I_{i}(x, y)}{\sum_{j=1}^{S} I_{i}(x, y)},
$$

where $i$ represents the respective channel, $S$ is the channel's number used by the image, generally $S=3$ in the space color RGB. The equation (6) describes Multi-scale with color correction algorithm:

$$
R_{M S R C R_{i}}(x, y)=C_{i}(x, y) R_{M S R_{i}}(x, y),
$$

where $C_{i}(x, y)$ is defined in equation (7):

$$
C_{i}(x, y)=f\left[I^{\prime}(x, y)\right] .
$$


The MSRCR provides the necessary color restoration, eliminating the color distortions and gray zones in the MSR output.

\section{CIELab Color Space Evaluation Method}

The evaluation methods used to measure image quality are closely related, in comparison to the original version or under some ideal image criteria proposed in the literature; the image distortion quantification is required in many image-processing fields. Color quantification incorporates psycho-physic elements related to human perception becoming more complex in a computing evaluation. However, the evaluation of the human vision system criteria has better results compared with image distortion evaluations [8].

Is used the CIELab color space to evaluate Retinex models, mainly because of its perception uniformity. CIELab Color space sets that for observe colors, they must be observed under a background going from white to grey with an standard $\mathrm{D}_{65}$ type illumination source which has similar features with the mid-day light, and with a temperature of $6,504{ }^{\circ} \mathrm{K}[8]$. Every source with these features is called $\mathrm{D}_{65}$. CIELab is the chromatic model used for color description in the human eye perception. This model is represented by three parameters, color lightness $(L)$, position between red and green color $(a)$, and the position between yellow and blue color $(b)$ [9]. Vectors $a$ and $b$ are calculated as shown in equation (8) [8]:

$$
\begin{gathered}
a=\left[C_{1}-C_{2} / 11\right]=\left[R_{a}-12 G_{a} / 11+B_{a} / 11\right], \\
b=(1 / 2)\left[C_{2}-C_{1}+C_{1}-C_{3}\right] /(4.5)=(1 / 9)\left[R_{a}+C_{2}+2 B_{a}\right],
\end{gathered}
$$

The CIELab chromaticity vector is represented by the magnitude of the distance between vectors $a$ and $b$. This indicates that for a high chromaticity value, the image color is more intense or saturated. Therefore, the average chromaticity present in the image processed will be better [10]. The average value of every one of the pixels where chromaticity were computed, represents a vector computed in equation (9):

$$
C_{M N}=\sum \sqrt{a_{x y}^{2}+b_{x y}^{2}} / M N
$$

\section{$4 \quad$ Results}

In the subjective criteria results obtained in the original image as environmental pollution produces gray areas and image processing with Simple Retinex and Multi-scale algorithms, the light source is identified and can be corrected only lighting, while the algorithm MSRCR, corrected lighting and color correction and thus can have better visibility through air pollution.

To do an image evaluation, is required a transformation in the RGB to CIELab color space, and the average chromaticity of the image is calculated. The best result is indicated by the highest value obtained.

In the Fig. 1 can be saw the objective and subjective results for each image with environmental pollution, being processed with the Single Retinex, MSR, and MSRCR models. The chromaticity computing evaluation for each image is performed using 
Chromatic Improvement of Backgrounds Images Captured with Environmental Pollution Using ...

equation (8), the first step is to transform the RGB color space into the CIELAB color space, and use the $a$ and $b$ CIELab components, for every pixel, to obtain the average values agree to the number of values in a $M \times N$ image dimensions $(M, N)$, where $M$ is the number of rows and $N$ is the number of columns.

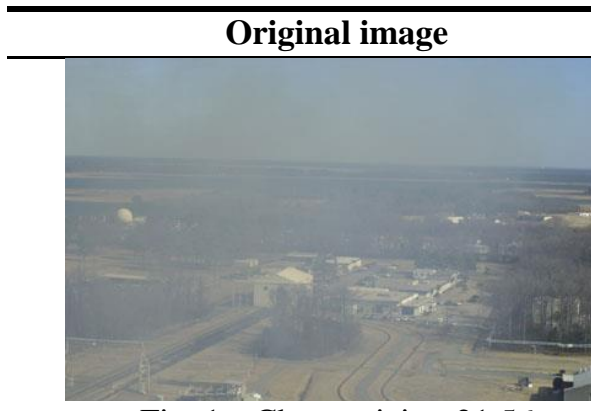

Fig. 1a. Chromaticity: 21.56

\section{Simple Retinex}

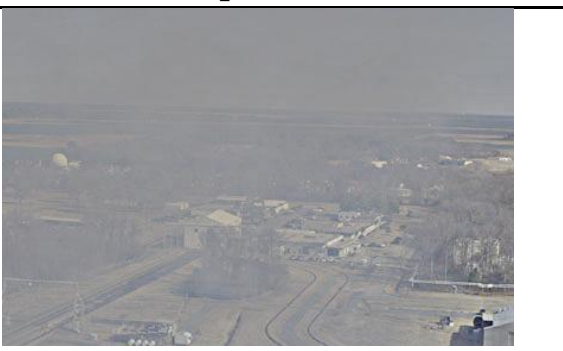

Fig. 1b. Chromaticity: 21.88

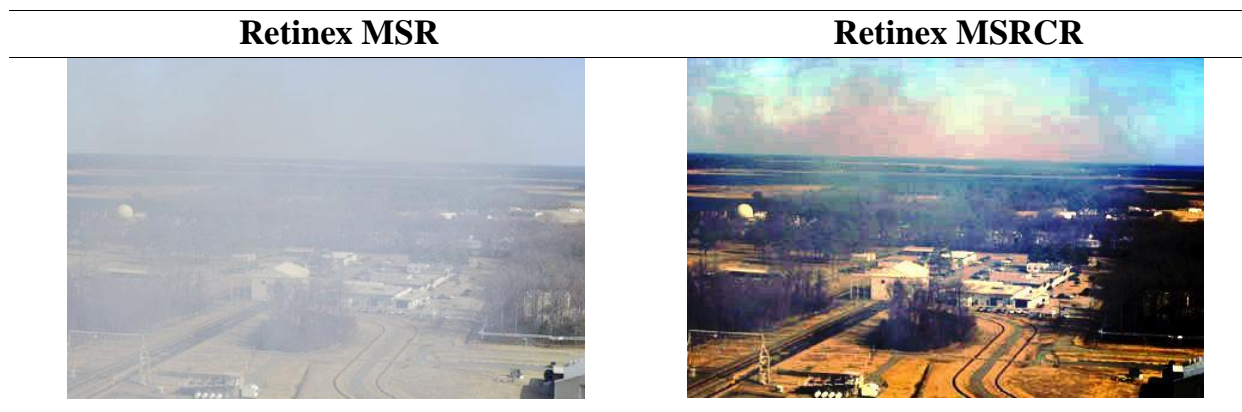

Fig. 1c. Chromaticity: 21.59

Fig. 1d. Chromaticity: 22.27

Fig. 1. Fig. 1a was taken with pollution, the other ones increases in chromaticity intensities as shown in Figs. 1b and 1c, where the best result is given by the analyzed MSRCR algorithm shown in Figure 1d.

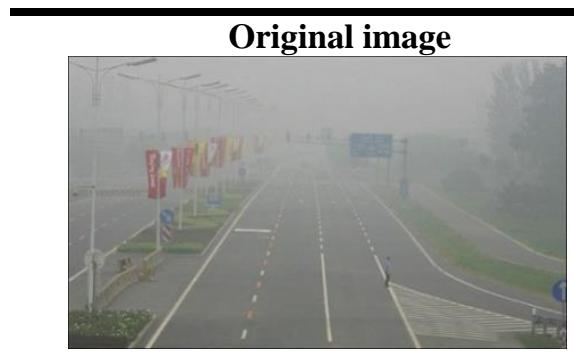

Fig. 2a. Chromaticity: 22.06

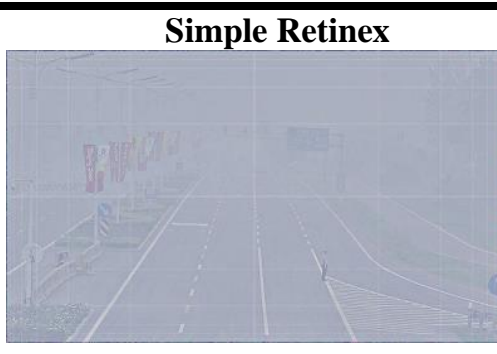

Fig. 2b. Chromaticity: 21.26 


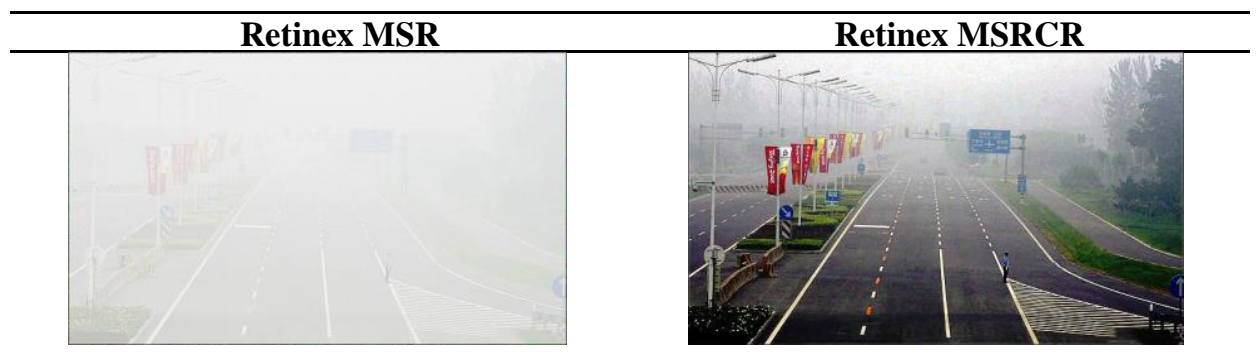

Fig. 2c. Chromaticity: 22.03

Fig. 2d. Chromaticity: 22.25

Fig. 2. Fig. 2a was taken with foggy, and it was processed by the Simple and multiscale Retinex algorithms ( $2 \mathrm{~b}$ and $2 \mathrm{c}$ ), it reduces the chromaticity levels of the image, contrary happens with the Retinex MSRCR where chromaticity values increases.

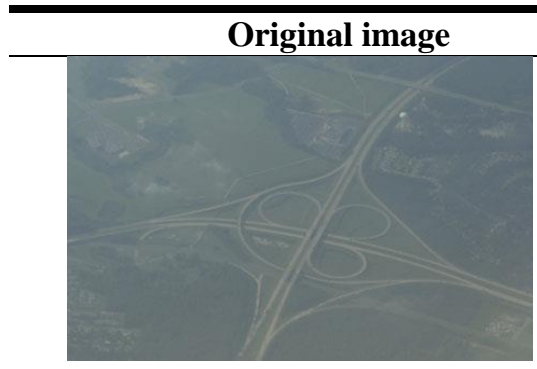

Fig. 3a. Chromaticity: 21.34

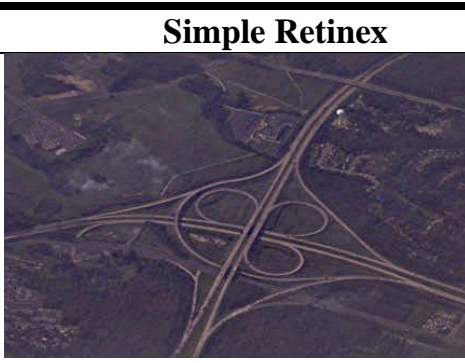

Fig. 3b. Chromaticity: 21.92

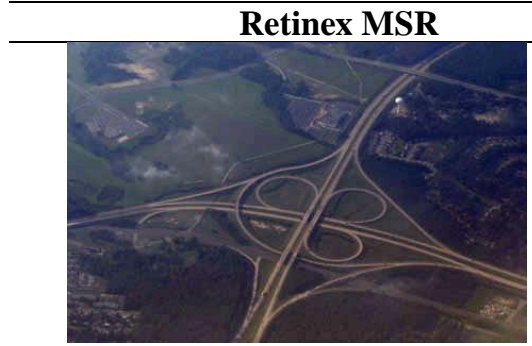

Fig. 3c. Chromaticity: 21.52

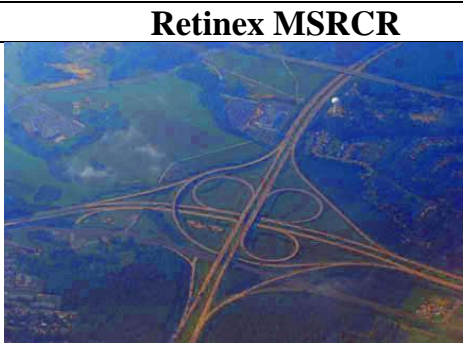

Fig. 3d. Chromaticity: 22.95

Fig. 3. Fig. 3a is an aerial photograph taken with pollution, and the image was processed by the Simple and multiscale Retinex algorithms, highest image chroma is processed by the MSRCR algorithm. 


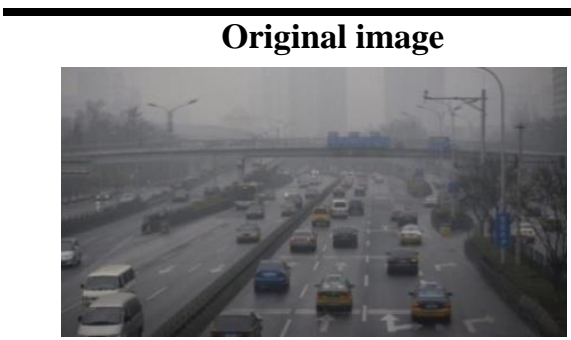

Figure 4a. Chromaticity: 22.34

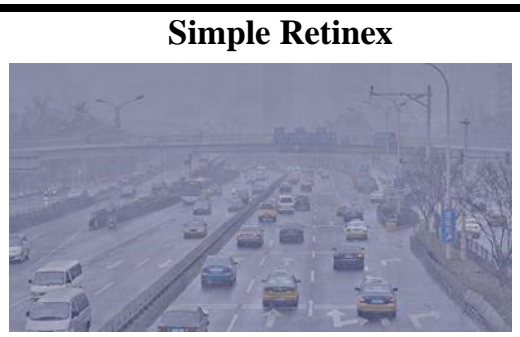

Figure 4b. Chromaticity: 20.99

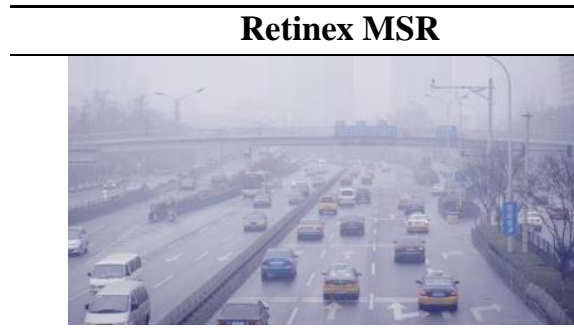

Figure 4c. Chromaticity: 21.95

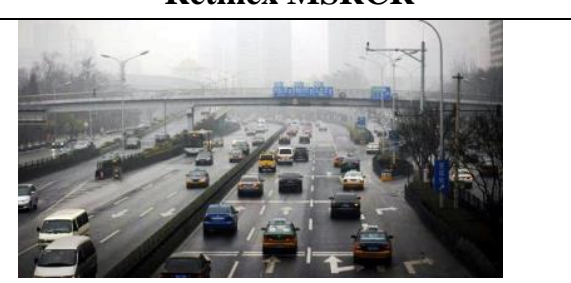

Figure 4d. Chromaticity: 22.59

Fig. 4. Fig. 4a was taken with pollution, processing results with Simple and MSR Retinex algorithms not improve the content of chromaticity, in Fig. 4d is observed the improved chromaticity content.

It is observed that the values obtained from the color evaluation methods, the Retinex model is efficient in chromaticity correction having better results in contrasting colors, these images corrupted with foggy and pollution are characterized as images affected in their pitch illuminant and wavelength content to capture air pollution.

\section{Conclusions}

To improve chromaticity content of the images captured in environments with air pollution, the Retinex model can be a tool that will serve to make corrections in the contrast of the images. In the results we can see that the efficiency of the Retinex model is directly related to the content of wavelengths that could be captured in digital imaging and because these vary the results that may be misinterpreted to be processed with a particular Retinex method.

Retinex can be applied to images with pollution as a tool that makes the correction of contrast colors, the performance of Retinex depend on the density of pollution, causing the captured colors do not match the colors obtained under a type lighting $\mathrm{D}_{65}$.

Acknowledgements. The authors would thank to the Instituto Politécnico Nacional de México and the CONACyT for their support in the realization of this research work. 


\section{References}

1. E. H. Land, J. J. McCann: Lightness and Retinex Theory. Journal of the Optical Society of America (1971)

2. G. Hines, U. Rahman, G. Woodell: Single-Scale Retinex Using Digital Signal Processor. Global Signal Processing Expo (GSPx) (2004)

3. J. M. Morel, A. B. Petro, C. Sbert: Fast implementation of color constancy algorithms. SPIE, vol. 7241 (2009)

4. D. J. Jobson, Z. Rahman: A multiscale Retinex for Bridging the gap between color images and the human observation of scenes. IEEE Transactions on image processing, $n^{\circ} 7$ (1997)

5. A. Rizzi, C. Gatta: From Retinex to Automatic Color Equalization: issues in developing a new algorithm for unsupervised color equalization. Journal of Electronic Imaging (2004)

6. J. McCann, B. Funt: Retinex in MATLAB. Journal of Electronic Imaging, $n^{\circ}$ 13, (2004)

7. G. Buchsbaum: A spatial processor model for object colour perception. Journal of the Franklin Institute (1980)

8. X. Zhang, B. A. Wandell: Colour image fidelity metrics evaluated using image distortion maps. Imaging Science and Techology Program, Department of Psychology (1998)

9. J. Schanda: Colorimetry: Understanding the CIE System. Wiley-Interscience (2007)

10. M. D. Fairchild: Color Appearance Models. Second Edition, John Wiley \& Sons, Ltd (2005)

11. V. Tsagaris, G. Ghirstoulas, V. Anastassopoulos: A Measure for Evaluation of the Information Content in color images. IEEE Inter. Conf. Imag. Process. (2005) 